\title{
A Predictor of Positive Drug Provocation Testing in Individuals With Saddle-Back Type ST-Segment Elevation
}

\author{
Kenji Shimeno, MD; Masahiko Takagi, MD; Keiko Maeda, MD; Hiroaki Tatsumi, MD; \\ Atsushi Doi, MD; Eiichiro Nakagawa, MD; Minoru Yoshiyama, MD
}

\begin{abstract}
Background: A drug provocation test is performed to unmask a type 1 electrocardiogram (ECG) in individuals with saddle-back type ST-segment elevation in the right precordial leads (SB-ECG). The study investigated predictors of positive responders (PR) by drug testing.

Methods and Results: A total of 58 consecutive individuals with SB-ECG in lead V2 were enrolled and drug testing was performed. In leads V2 at standard and the third intercostal space (V2IC3), the QRS duration was measured, the amplitudes of r' wave (r'), ST-segment 20 and $40 \mathrm{~ms}$ after the r' wave (r'20 and r' 40 , respectively), the bottom of the ST-segment (STb), the differences between r' and r'20 (r'-r'20), r' and r'40 (r'-r'40), r' and STb $\left(\mathrm{r}^{\prime}-\mathrm{ST} \mathrm{b}\right)$, and the descending rate of the ST-segment [(r'-r'20)/r'; DR20, (r'-r'20)/(r'-STb); DR20-STb] were also measured. Thirty-five PR had significantly longer QRS duration, larger r'20 and r'40, and smaller r'-r'20, DR20, and DR20-STb than negative responders. DR20 and DR20-STb in leads V2 and V2IC3 were most significantly different between 2 groups. The positive and negative predictive values of 'DR20-STb in lead V2IC3 <0.62' for prediction of positive tests were $92.3 \%$ and $81.8 \%$, respectively.
\end{abstract}

Conclusions: In individuals with SB-ECG, DR20 and DR20-STb in leads V2 and V2IC3 might be useful predictors of positive testing. (Circ J 2009; 73: 1836-1840)

Key Words: Brugada syndrome; Drug provocation testing; Electrocardiogram; ST segment

B rugada syndrome is characterized by a ST-segment elevation in the right precordial leads and associated with sudden cardiac death secondary to rapid polymorphic ventricular tachycardia or ventricular fibrillation. ${ }^{1}$ A recent consensus report proposed 3 types of ST-segment elevation (types 1 to 3 ) and indicated that a coved-type ST-segment elevation $>0.2 \mathrm{mV}$ (type 1 electrocardiogram (ECG)), in the presence or absence of $\mathrm{Na}$ channel blockers, is necessary for diagnosis of this syndrome. ${ }^{2,3}$ Individuals with saddle-back type ST-segment elevation in the right precordial leads (SB-ECG), which applies to type 2 or $3 \mathrm{ECG}$, are much more prevalent than those with type $1 \mathrm{ECG},{ }^{4}$ at a rate of 58/10,000 inhabitants in Japan. ${ }^{5}$ Although previous reports show asymptomatic individuals with SB-ECG who exhibit a type 1 ECG under drug administration are low risk for fatal cardiac event for middle-term periods, ${ }^{6,7}$ long-term prognosis in those individuals is still unclear. For individuals with SB-ECG, therefore, a drug provocation test using Na channel blockers is necessary and generally considered helpful to diagnose a type 1 ECG.6,8,9 In some cases, the drug testing is accompanied with the risk of drug-induced ventricular tachyarrhythmias. ${ }^{3,10}$ It is difficult to undergo drug provocation testing for all individuals with SB-ECG because of the high prevalence. Although the differences between baseline ECG parameters of positive responders (PR) and negative responders (NR) on the drug testing have been reported, ${ }^{11-13}$ it remains unclear which parameters on baseline ECG are clinically useful as predictors of test outcome. However, it has been reported that the ECG recorded at higher intercostal space is more sensitive and useful for diagnosis of Brugada syndrome than that recorded at the standard position. ${ }^{14-19}$

In the present study, to avoid the risk of drug-induced ventricular tachyarrhythmias on drug testing, we analyzed baseline ECG parameters recorded at the standard position and the third intercostal space in individuals with SB-ECG, and evaluated predictors of PR on drug provocation testing.

\section{Methods}

In a total of 62 consecutive individuals with SB-ECG, we performed the drug provocation test using pilsicainide, a class Ic pure Na channel blocker, between September 2002 and December 2007. In these individuals, SB-ECG in leads $\mathrm{V} 1$ and V2 was exhibited in 4 and 58 individuals, respectively. In this study, we enrolled 58 individuals (55 males, mean age $51 \pm 11$ years) with SB-ECG in lead V2. Of the 58 individuals, 48 individuals showed SB-ECG in lead V2 at the third intercostals space (V2IC3). The remaining 10 individuals did not show type 1 ECG in lead V2IC3. Patients who had exhibited a diagnostic ECG (type 1 ECG) at any

(Received April 28, 2009; revised manuscript received June 24, 2009; accepted June 25, 2009; released online September 7, 2009)

Department of Internal Medicine and Cardiology, Osaka City University Graduate School of Medicine, Osaka, Japan

Mailing address: Masahiko Takagi, MD, Department of Internal Medicine and Cardiology, Osaka City University Graduate School of Medicine, 1-4-3 Asahimachi, Abeno-ku, Osaka 545-8585, Japan. E-mail: m7424580@ msic.med.osaka-cu.ac.jp

All rights are reserved to the Japanese Circulation Society. For permissions, please e-mail: cj@j-circ.or.jp 

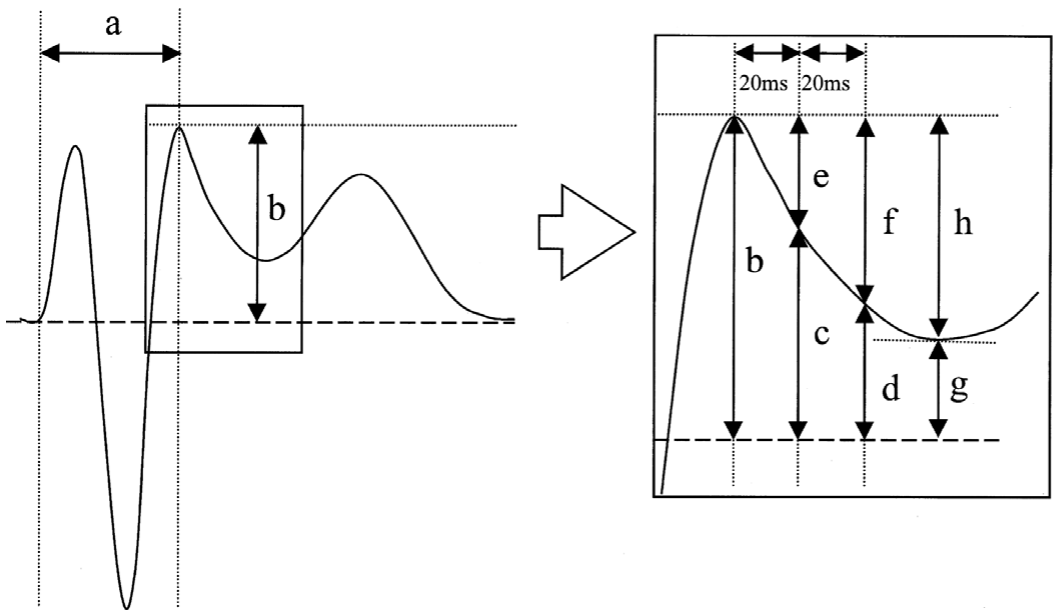

a; QRS duration

$\mathrm{b} ; \mathrm{r}$ '

c; r'20

d; r'40

e; r'-r'20

f; r'-r' 40

$\mathrm{g} ; \mathrm{ST}_{\mathrm{b}}$

$\mathrm{h} ; \mathrm{r}^{\prime}-\mathrm{ST}_{\mathrm{b}}$

DR20; e/b

$\mathrm{DR} 20-\mathrm{ST}_{\mathrm{b}} ; \mathrm{e} / \mathrm{h}$

Figure 1. The measurement parameters in leads V2 and V2IC3. r', amplitude of the peak of r' wave. r'20, r'40, amplitudes of ST-segment $20 \mathrm{~ms}$ and $40 \mathrm{~ms}$ after the r' wave. STb, amplitude of the bottom of the ST-segment. r'-r'20, r'-r'40, r'-STb, differences in amplitudes between r' and r'20, r' and r'40, and r' and STb, respectively. DR20 and DR20-STb, rate of descent of the ST-segment [(r'-r'20)/r' and (r'-r'20)/(r'-STb), respectively].

Table 1. Patient Characteristics

\begin{tabular}{lccc}
\hline & $\begin{array}{c}\text { Positive responders } \\
(\mathrm{n}=35)\end{array}$ & $\begin{array}{c}\text { Negative responders } \\
(\mathrm{n}=23)\end{array}$ & P value \\
\hline Age (years) & $50.2 \pm 9.0$ & $50.9 \pm 12.7$ & 0.94 \\
Gender (male) & 34 & 21 & 0.56 \\
Type 2 electrocardiogram & 32 & 20 & 0.67 \\
Type 3 electrocardiogram & 3 & 3 & 0.67 \\
Symptomatic patients & 3 & 0 & 0.27 \\
Family history of sudden cardiac death & 5 & 2 & 0.69 \\
\hline
\end{tabular}

time in the absence of $\mathrm{Na}+$ channel blockers at the standard position were excluded from this study. In all individuals, physical examination, chest X-p, and hematological testing yielded no abnormal findings, and no evidence of structural heart disease was detected on echocardiography. Three patients were symptomatic. One had a history of syncope, and 2 had survived sudden death. The remaining 55 individuals were asymptomatic. Seven individuals had a family history of sudden death (at less than 45 years of age). Fiftytwo individuals showed a type 2 ECG, the other 6 individuals showed a type 3 ECG.

Drug provocation testing was performed with intravenous pilsicainide $(1 \mathrm{mg} / \mathrm{kg}, 5 \mathrm{mg} / \mathrm{min})$ in a quiet room equipped for cardiopulmonary resuscitation. Twelve-lead ECG at the standard position and precordial ECG at the third intercostal space were simultaneously recorded on a computer-based digital recorder system (CardioLab system) before and after the administration of pilsicainide. A positive response was defined as the occurrence of type 1 ECG at the standard position. The infusion was terminated when a positive response was elicited or major adverse events occurred, defined as ventricular arrhythmias or important conduction slowing (prolongation of QRS $>30 \%$ of basal value).

In all 58 individuals, the following parameters in lead V2 $(n=58)$ and V2IC3 $(n=48)$ were analyzed on the baseline ECG: heart rate (HR), QRS duration (QRSv2), the amplitude of the peak of r' wave (r'), the amplitudes of the STsegment $20 \mathrm{~ms}$ and $40 \mathrm{~ms}$ after the r' wave (r'20 and r' 40 , respectively), and the bottom of the ST-segment $(\mathrm{STb})$. We calculated the differences in amplitudes between r' and r'20 (r'-r'20), r' and r'40 (r'-r'40), r' and STb (r'-STb), and 2 types of the descending rate of the ST-segment; (1) DR20; the number of r'-r'20 divided by r' [(r'-r'20)/r'] and (2) DR20-STb; the number of r'-r'20 divided by r'-STb $\left[\left(r^{\prime}-r^{\prime} 20\right) /\left(r^{\prime}-S T b\right)\right]$. In addition, the $S$ wave duration in lead II and QRS duration in lead V5 (QRSv5) were measured. The method of measurement in leads V2 and V2IC3 is shown in Figure 1. All these parameters were measured at a paper speed of $100 \mathrm{~mm} / \mathrm{s}$ with CardioLab software (GE Medical Systems) on a HP workstation Xw8000 computer. All measured data were collected from 3 consecutive ECGs and averaged. Results were compared between PR and NR.

Quantitative values in all ECG parameters are expressed as the mean \pm standard deviation values. For comparisons between PR and NR, the non-parametric Mann-Whitney U-test was used. All tests were 2-sided, and statistical significance was defined as a value of $\mathrm{P}<0.05$. For prediction of test results, sensitivity, specificity, positive predictive value (PPV), and negative predictive value (NPV) were used.

\section{Results}

Patient characteristics in the 2 groups are shown in Table 1. There were 35 PR patients, while the remaining 23 individuals were NR. No significant adverse events occurred. All 3 symptomatic patients and 5 of the 7 individuals with a family history of sudden cardiac death were PR.

Baseline ECG parameters are shown in Table 2. In lead V2, compared with NR, PR exhibited significantly longer QRS duration (90.9 \pm 9.1 vs $85.1 \pm 6.1 \mathrm{~ms} ; \mathrm{P}=0.01$, PR vs 
Table 2. Measured Parameters on Baseline ECG in Leads V2 and V2IC3

\begin{tabular}{|c|c|c|c|c|c|c|}
\hline & \multicolumn{3}{|c|}{ V2 } & \multicolumn{3}{|c|}{ V2IC3 } \\
\hline & $\begin{array}{l}\text { Positive } \\
\text { responders }\end{array}$ & $\begin{array}{l}\text { Negative } \\
\text { responders }\end{array}$ & $P$ value & $\begin{array}{l}\text { Positive } \\
\text { responders }\end{array}$ & $\begin{array}{l}\text { Negative } \\
\text { responders }\end{array}$ & $P$ value \\
\hline $\mathrm{n}$ & 35 & 23 & & 28 & 20 & \\
\hline HR (beats/min) & $59.4 \pm 9.3$ & $64.8 \pm 12.4$ & 0.16 & & & \\
\hline QRS duration (ms) & $90.9 \pm 9.1$ & $85.1 \pm 6.1$ & 0.01 & $90.9 \pm 9.2$ & $84.6 \pm 6.7$ & 0.03 \\
\hline $\mathrm{r}^{\prime}(\mathrm{mV})$ & $0.38 \pm 0.14$ & $0.42 \pm 0.20$ & 0.45 & $0.50 \pm 0.24$ & $0.53 \pm 0.22$ & 0.42 \\
\hline r'20 (mV) & $0.28 \pm 0.10$ & $0.23 \pm 0.11$ & 0.03 & $0.32 \pm 0.15$ & $0.22 \pm 0.11$ & $<0.01$ \\
\hline $\mathrm{r}^{\prime} 40(\mathrm{mV})$ & $0.22 \pm 0.07$ & $0.18 \pm 0.08$ & 0.02 & $0.21 \pm 0.08$ & $0.15 \pm 0.07$ & $<0.01$ \\
\hline r'-r'20 (mV) & $0.10 \pm 0.08$ & $0.19 \pm 0.13$ & $<0.001$ & $0.18 \pm 0.12$ & $0.31 \pm 0.16$ & $<0.01$ \\
\hline r'-r'40 (mV) & $0.16 \pm 0.11$ & $0.25 \pm 0.18$ & 0.02 & $0.29 \pm 0.19$ & $0.38 \pm 0.23$ & 0.15 \\
\hline $\mathrm{STb}(\mathrm{mV})$ & $0.18 \pm 0.07$ & $0.17 \pm 0.08$ & 0.31 & $0.14 \pm 0.06$ & $0.12 \pm 0.07$ & 0.09 \\
\hline r'-STb (mV) & $0.20 \pm 0.13$ & $0.25 \pm 0.18$ & 0.27 & $0.36 \pm 0.24$ & $0.41 \pm 0.24$ & 0.27 \\
\hline DR20 & $0.24 \pm 0.14$ & $0.43 \pm 0.15$ & $<0.0001$ & $0.34 \pm 0.14$ & $0.57 \pm 0.16$ & $<0.0001$ \\
\hline $\mathrm{DR} 20-\mathrm{ST} \mathrm{b}$ & $0.50 \pm 0.11$ & $0.80 \pm 0.12$ & $<0.0001$ & $0.51 \pm 0.12$ & $0.78 \pm 0.12$ & $<0.0001$ \\
\hline
\end{tabular}

Significant at 0.05 level.

V2IC3, V2 at the third intercostal space; HR, heart rate; r', amplitude of the peak of r' wave; r'20, r'40, amplitudes of ST-segment $20 \mathrm{~ms}$ and $40 \mathrm{~ms}$ after the r' wave; r'-r'20, r'-r'40, r'-STb, differences in amplitudes between r' and r'20, r' and r'40, and r' and STb, respectively; STb, amplitude of the bottom of the ST-segment; DR20, DR20-STb, rate of descent of the ST-segment (r'-r'20/r' and r'-r'20/r'-STb, respectively).

a) positive responder

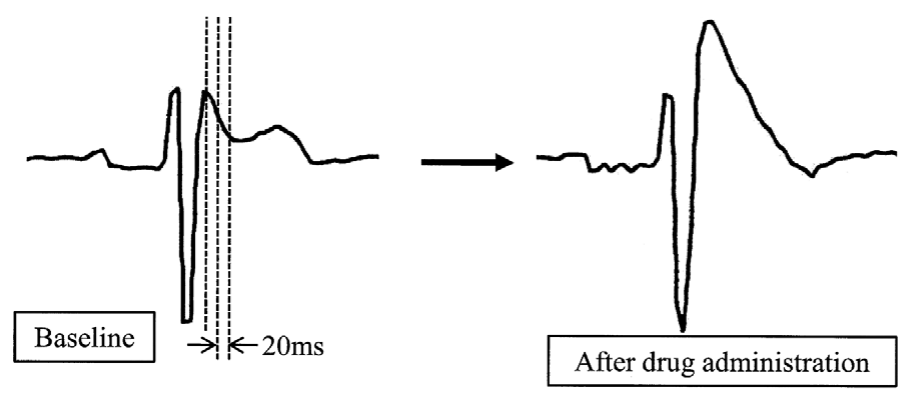

b) negative responder

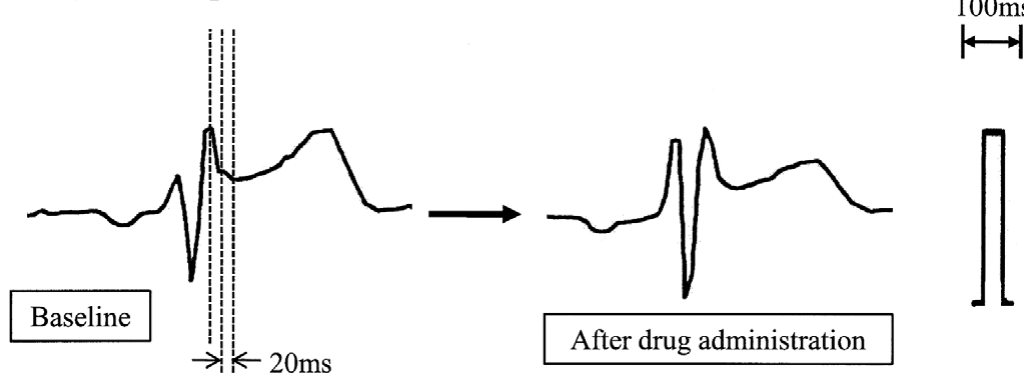

Figure 2. Representative ECGs of positive and negative responders in lead V2IC3. QRS duration, r', r'20, r'40, r'-r'20, DR20, and DR20-STb were $93 \mathrm{~ms}, 0.42 \mathrm{mV}, 0.30 \mathrm{mV}, 0.20 \mathrm{mV}, 0.12 \mathrm{mV}, 0.29$, and 0.47 , respectively, in the positive responder (a), and $94 \mathrm{~ms}, 0.42 \mathrm{mV}, 0.19 \mathrm{mV}, 0.16 \mathrm{mV}$, $0.23 \mathrm{mV}, 0.55$, and 0.92 , respectively, in the negative responder (b). V2IC3; V2 at the third intercostal space. Other abbreviations as Figure 1.

NR), larger value of r' 20 and r'40 $(0.28 \pm 0.10$ vs $0.23 \pm$ $0.11 \mathrm{mV} ; \mathrm{P}=0.03,0.22 \pm 0.07$ vs $0.18 \pm 0.08 \mathrm{mV} ; \mathrm{P}=0.02$, PR vs NR, respectively), and significantly smaller value of r'-r'20, r'-r'40, DR20, and DR20-STb $(0.10 \pm 0.08$ vs $0.19 \pm$ $0.13 \mathrm{mV} ; \mathrm{P}<0.001,0.16 \pm 0.11$ vs $0.25 \pm 0.18 \mathrm{mV} ; \mathrm{P}=0.02$, $0.24 \pm 0.14$ vs $0.43 \pm 0.15 ; \mathrm{P}<0.0001,0.50 \pm 0.11$ vs $0.80 \pm 0.12$; $\mathrm{P}<0.0001$, PR vs NR, respectively). Neither r' nor STb differed significantly between the 2 groups (Table 2). Moreover, in lead V2IC3, similar results are obtained (Table 2). Representative ECGs are shown in Figure 2. The $\mathrm{S}$ wave duration in lead II and the difference between QRSv2 and QRSv5 (QRSv2-QRSv5), were not significantly different between PR and NR $(30.4 \pm 20.5$ vs $35.9 \pm 18.6 \mathrm{~ms} ; \mathrm{P}=0.59$, $-5.8 \pm 8.9$ vs $-7.4 \pm 12.1 \mathrm{~ms} ; \mathrm{P}=1.00$, PR vs $\mathrm{NR}$, respectively).

Of these parameters, DR20 and DR20-STb in leads V2 and V2IC3 differed most significantly between the 2 groups
(Figure 3). We plotted receiver operating characteristic curves for these parameters, and determined the point exhibiting the highest sensitivity and specificity. Sensitivity, specificity, PPV, and NPV using these parameters with the best cut-off point are shown in Table 3. All of the 4 parameters could predict the test outcome well. In particular, DR20-STb in lead V2IC3 was the most useful predictor in these parameters. When 'DR20-STb in lead V2IC3 $<0.62$ ' was tested as a predictor of positive outcome, parameters for the prediction of positive outcome on drug testing were as follows: sensitivity $85.7 \%$, specificity $90.0 \%$, PPV $92.3 \%$, and NPV $81.8 \%$.

\section{Discussion}

In the present study, we evaluated the differences in baseline ECG characteristics between PR and NR in individuals 

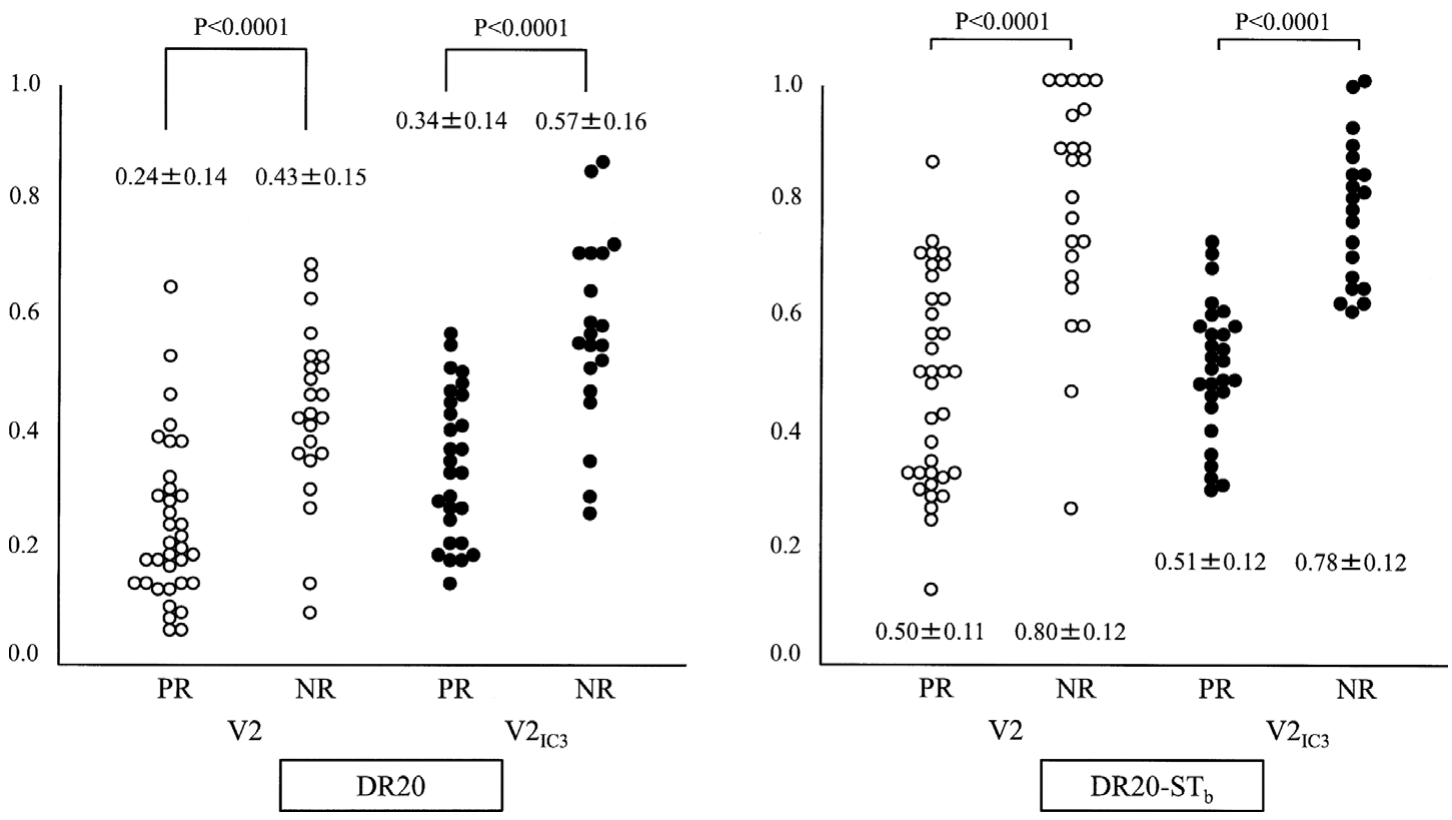

Figure 3. Comparison of DR20 and DR20-STb values in leads V2 and V2IC3 between PR and NR. DR20 and DR20-STb in leads V2 and V2IC3 were significantly different between the 2 groups $(\mathrm{P}<0.0001)$. PR, positive responders; NR, negative responders. Other abbreviations as Figure 1.

Table 3. Parameters for the Prediction of Positive Outcome on Drug Provocation Testing

\begin{tabular}{lccccc}
\hline Parameters & Cut-off & Sensitivity & Specificity & PPV & NPV \\
\hline DR20 (V2) & $<0.35$ & 80.0 & 82.6 & 87.5 & 73.1 \\
DR20-STb (V2) & $<0.63$ & 77.1 & 82.6 & 87.1 & 70.4 \\
DR20 (V2IC3) & $<0.47$ & 82.1 & 80.0 & 85.2 & 76.2 \\
DR20-STb (V2IC3) & $<0.62$ & 85.7 & 90.0 & 92.3 & 81.8 \\
& $<0.60$ & 82.1 & 100.0 & 100.0 & 80.0 \\
\hline
\end{tabular}

PPV, positive predictive value; NPV, negative predictive value; V2IC3, V2 at the third intercostal space.

with SB-ECG on drug provocation testing. The results of the present study show that DR20 and DR20-STb in leads V2 and V2IC3 are useful non-invasive predictors of positive outcome on drug testing.

We showed the values of QRS duration, r'20, and r'40 were significantly larger and those of r'-r'20, DR20, and DR20-STb were significantly smaller in PR, compared with those in NR. DR20 and DR20-STb, in particular, differed most significantly between the 2 groups. These findings indicate that PR with SB-ECG exhibited a 'gradually' descending ST-segment, like type 1 ECG, on the baseline ECG. The cellular mechanism of type 1 ECG has been found to involve the following: incomplete depolarization during phase 0 produces a deep notch in phase 1 of the epicardial action potential, which augments a transmural current from the endocardium and creates a prominent $\mathbf{J}$ point (ST-segment) elevation. The subsequent dome produces an attenuated transmural current flow that contributes to a downslope (coved-type) of the ST segment. ${ }^{20-23} \mathrm{We}$ speculate that PR with SB-ECG might have the same electrophysiological characteristics, arising slowly from a deep notch in phase 1 of epicardial action potential as patients with type 1 ECG, and manifest some of them on the baseline ECG.

In this study, DR20 and DR20-STb in lead V2 and V2IC3 could predict the test outcome well. In particular, DR20$\mathrm{STb}$ in lead V2IC3 was the most useful predictor in these parameters. Some previous studies have shown that the ECG recorded at a higher intercostal space is more useful for the diagnosis of Brugada syndrome than that recorded at standard position. ${ }^{14-19}$ In the present study, we support the usefulness of the ECG at the third intercostal space to evaluate predictors of PR on the drug testing.

Several predictors of positive testing have been reported. Meregalli et al reported the QRS duration, the J-point elevation (leads V1, V2, V1IC3, and V2IC3), and the S wave duration in lead II on baseline ECG were significantly different between PR and NR. However, they failed to predict the outcome of testing, using these parameters. ${ }^{11} \mathrm{In}$ the present study, only QRS duration in leads V2 and V2IC3 was significantly different between PR and NR, while r' in leads V2 and V2IC3, and the S wave duration in lead II were not significantly different. Nakazawa et al reported that the difference of the QRS duration between in leads V2 and V5 (QRSv2-QRSv5) $\geq 0 \mathrm{~ms}$ was found to be a predictor of positive drug provocation test. ${ }^{12}$ In the current study, however, this predictor was not significantly different between the 2 groups. Hermida et al reported that baseline $\mathrm{J}$ wave elevation $>0.16 \mathrm{mV}$ in lead V2 at the second intercostal space had a specificity of $100 \%$, a sensitivity of $40 \%$, a PPV of $100 \%$ and a NPV of $28 \% .{ }^{13}$ Comparing these reports, DR20 and DR20-STb in lead V2 and V2IC3 in the present study are better predictors of positive drug testing.

The non-invasive ECG parameters, DR20 and DR20-STb 
in leads V2 and V2IC3, might be useful for distinguishing PR from NR on drug provocation testing. These ECG parameters might make drug provocation testing unnecessary for unmasking a type $1 \mathrm{ECG}$, and consequently avoid the risk of drug-induced ventricular tachyarrhythmias. Moreover, we could discriminate non-invasively the patients with arrhythmogenic substrate in Brugada Syndrome from the individuals without it.

The present study has several limitations. First, DR20 or DR20-STb was calculated with r', r'20, and STb. However, the visual measurement of r' 20 on the ECG at a speed of $25 \mathrm{~mm} / \mathrm{s}$ is difficult. In this study, we magnified the ECGs and measured with a CardioLab system as digital data. For measurement of r'20 using a standard ECG recording system, it might be necessary to record at a high paper speed and use magnification. If these parameters can be measured in an automated ECG analysis system, these might be more useful in the clinical setting. Second, it is known that the electrocardiographic features of Brugada syndrome are dynamic and often concealed. ${ }^{24,25}$ Therefore, judgment regarding the presence of this syndrome with the ECG at a single recording is likely to underestimate the diagnosis of it. It might be necessary to record ECGs repeatedly in the same individuals for evaluation of this matter. Third, it has been known that some individuals showed type 1 ECG only in the higher intercostal space on drug testing. However, it is still controversial whether the results of testing should be judged by the ECG involving in the higher intercostal space, because of concern about falsepositive results as the recent consensus report showed. ${ }^{2}$ Therefore, we defined the positive response as the occurrence of type 1 ECG only at the standard position. Finally, the present study is retrospective evaluation. Therefore, further prospective study is needed to assess the usefulness of these non-invasive ECG parameters as predictors of positive drug provocation test.

\section{Conclusion}

In individuals with saddle-back type ST-segment elevation, DR20 and DR20-STb in leads V2 and V2IC3 might be useful as non-invasive predictors of positive pilsicainide testing. These parameters could discriminate the patients with arrhythmogenic substrate in Brugada syndrome from the individuals without it.

\section{References}

1. Brugada P, Brugada J. Right bundle branch block, persistent ST segment elevation and sudden cardiac death: A distinct clinical and electrocardiographic syndrome: A multicenter report. J Am Coll Cardiol 1992; 20: 1391-1396.

2. Antzelevitch C, Brugada P, Borggrefe M, Brugada J, Brugada R, Corrado D, et al. Brugada syndrome: Report of the second consensus conference: Endorsed by the Heart Rhythm Society and the European Heart Rhythm Association. Circulation 2005; 111: 659-670.

3. Wilde AA, Antzelevitch C, Borggrefe M, Brugada J, Brugada R, Brugada P, et al. Proposed diagnostic criteria for the Brugada syndrome: Consensus report. Circulation 2002; 106: 2514-2519.

4. Hermida JS, Lemoine JL, Aoun FB, Jarry G, Rey JL, Quiret JC. Prevalence of the Brugada syndrome in an apparently healthy population. Am J Cardiol 2000; 86: 91-94.

5. Miyasaka Y, Tsuji H, Yamada K, Tokunaga S, Saito D, Imuro Y, et al. Prevalence and mortality of the Brugada-type electrocardiogram in one city in Japan. J Am Coll Cardiol 2001; 38: 771-774.

6. Priori SG, Napolitano C, Gasparini M, Pappone C, Della Bella P, Giordano U, et al. Natural history of Brugada syndrome: Insights for risk stratification and management. Circulation 2002; 105: $1342-$ 1347.

7. Takagi M, Yokoyama Y, Aonuma K, Aihara N, Hiraoka M; Japan Idiopathic Ventricular Fibrillation Study (J-IVFS) Investigators. Clinical Characteristics and Risk Stratification in Symptomatic and Asymptomatic Patients with Brugada Syndrome: Multicenter Study in Japan. J Cardiovasc Electrophysiol 2007; 18: 1244-1251.

8. Brugada R, Brugada J, Antzelevitch C, Kirsch GE, Potenza D, Towbin JA, et al. Sodium channel blockers identify risk for sudden death in patients with ST-segment elevation and right bundle branch block but structurally normal hearts. Circulation 2000; 101: 510515.

9. Priori SG, Napolitano C, Gasparini M, Pappone C, Della Bella P, Brignole $\mathrm{M}$, et al. Clinical and genetic heterogeneity of right bundle branch block and ST-segment elevation syndrome: A prospective evaluation of 52 families. Circulation 2000; 102: 2509-2515.

10. Morita H, Morita ST, Nagase S, Banba K, Nishii N, Tani Y, et al. Ventricular arrhythmia induced by sodium channel blocker in patients with Brugada syndrome. J Am Coll Cardiol 2003; 42: $1624-$ 1631.

11. Meregalli PG, Ruijter JM, Hofman N, Bezzina CR, Wilde AA, Tan HL. Diagnostic value of flecainide testing in unmasking SCN5Arelated Brugada syndrome. J Cardiovasc Electrophysiol 2006; 17: $857-864$.

12. Nakazawa K, Sakurai T, Kishi R, Takagi A, Osada K, Ryu S, et al. Discrimination of Brugada syndrome patients from individuals with the saddle-back type ST-segment elevation using a marker of the standard 12-leadelectrocardiography. Circ J 2007; 71: 546-549.

13. Hermida JS, Denjoy I, Jarry G, Jandaud S, Bertrand C, Delonca J. Electrocardiographic predictors of Brugada type response during $\mathrm{Na}$ channel blockade challenge. Europace 2005; 7: 447-453.

14. Shimizu W, Matsuo K, Takagi M, Tanabe Y, Aiba T, Taguchi A, et al. Body surface distribution and response to drugs of ST segment elevation in Brugada syndrome: Clinical implication of eighty-sevenlead body surface potential mapping and its application to twelve-lead electrocardiograms. J Cardiovasc Electrophysiol 2000; 11: 396-404.

15. Nakazawa K, Sakurai T, Takagi A, Kishi R, Osada K, Miyazu O, et al. Clinical significance of electrocardiography recordings from a higher intercostals space for detection of the brugada sign. Circ $J$ 2004; 68: 1018-1022.

16. Hisamatsu K, Morita H, Fukushima K, Takenaka S, Nagase S, Nakamura K, et al. Evaluation of the usefulness of recording the ECG in the 3rd intercostal space and prevalence of Brugada-type ECG in accordance with recently established electrocardiographic criteria. Circ J 2004; 68: 135-138.

17. Sangwatanaroj S, Prechawat S, Sunsaneewitayakul B, Sitthisook S, Tosukhowong P, Tungsanga K. New electrocardiographic leads and the procainamide test for the detection of the Brugada sign in sudden unexplained death syndrome survivors and their relatives. Eur Heart $J$ 2001; 22: 2290-2296.

18. Miyamoto K, Yokokawa M, Tanaka K, Nagai T, Okamura H, Noda T, et al. Diagnostic and prognostic value of a type 1 Brugada electrocardiogram at higher (third or second) V1 to V2 recording in men with Brugada syndrome. Am J Cardiol 2007; 99: 53-57.

19. Ueyama T, Shimizu A, Yamagata T, Esato M, Ohmura M, Yoshiga Y, et al. Different effect of the pure Na+ channel-blocker pilsicainide on the ST-segment response in the right precordial leads in patients with normal left ventricular function. Circ J 2007; 71: 57-62.

20. Yan GX, Antzelevitch C. Cellular basis for the electrocardiographic J wave. Circulation 1996; 93: 372-379.

21. Antzelevitch C. The Brugada syndrome. J Cardiovasc Electrophysiol 1998; 9: 513-516.

22. Yan GX, Antzelevitch C. Cellular basis for the Brugada syndrome and other mechanisms of arrhythmogenesis associated with ST-segment elevation. Circulation 1999; 100: 1660-1666.

23. Kurita T, Shimizu W, Inagaki M, Suyama K, Taguchi A, Satomi K, et al. The electrophysiologic mechanism of ST-segment elevation in Brugada syndrome. J Am Coll Cardiol 2002; 40: 330-334.

24. Mizumaki K, Fujiki A, Tsuneda T, Sakabe M, Nishida K, Sugao M, et al. Vagal activity modulates spontaneous augmentation of ST elevation in the daily life of patients with Brugada syndrome. J Cardiovasc Electrophysiol 2004; 15: 667-673.

25. Tatsumi H, Takagi M, Nakagawa E, Yamashita H, Yoshiyama M. Risk stratification in patients with Brugada syndrome: Analysis of daily fluctuations in 12-lead electrocardiogram (ECG) and signalaveraged electrocardiogram (SAECG). $J$ Cardiovasc Electrophysiol 2006; 17: 705-711. 